PATIENT SAFETY

M.D.I. Vergouwen

J. Fang

L.K. Casaubon

M.K. Kapral

M. Stamplecoski

A. Robertson

F.L. Silver, on behalf

of the Investigators of

the Registry of the

Canadian Stroke

Network

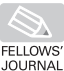

JOURNAL

\title{
A 5-Item Prediction Rule to Identify Severe Renal Dysfunction in Patients with Acute Stroke
}

BACKGROUND AND PURPOSE: Although patients with severe renal dysfunction who receive iodinated contrast are at high risk of CIN, contrast-enhanced CT scans are often obtained without prior knowledge of kidney function in patients with acute stroke. We aimed to develop a tool to identify patients with acute stroke at a high risk of $\mathrm{CIN}$ in the absence of a recent GFR.

MATERIALS AND METHODS: We used the RCSN (9872 patients) and OSA (2544 patients) for our derivation and validation cohort, respectively. A multivariable logistic regression model was performed to develop a predictive tool to identify severe renal dysfunction (defined as a GFR $<30 \mathrm{~mL} / \mathrm{min} / 1.73$ $\mathrm{m}^{2}$ ).

RESULTS: The overall prevalence of severe renal dysfunction was $4.9 \%$ and $5.2 \%$ in the derivation and validation cohort, respectively. The prediction rule was designed as follows: (age in years) + (5 points for women $)+(5$ points for history of diabetes mellitus $)+(15$ points for preadmission insulin use $)+$ (10 points for history of hypertension). The prevalence of severe renal dysfunction is negligible in patients with a total score of $\leq 70(\leq 0.005 \%-0.7 \%)$ but increases with higher Renal Risk Scores (eg, scores 71-80: 2.1\%-2.2\%; scores 91-100: 6.6\%-7.1\%; scores 111-120: 15.9\%-28.1\%).

concLusıons: The Renal Risk Score is a validated tool that helps clinicians select which patients with stroke can safely proceed to contrast-enhanced brain imaging without waiting for laboratory evidence of good renal function.

ABBREVIATIONS: $\mathrm{Cl}=$ confidence interval; $\mathrm{CIN}=$ contrast-induced nephropathy; $\mathrm{CKD}$-EPI = Chronic Kidney Disease Epidemiology Collaboration; GFR = glomerular filtration rate; IQR = interquartile range; OR = odds ratio; OSA = Ontario Stroke Audit; RCSN = Registry of the Canadian Stroke Network

$n$ patients presenting to the hospital with an acute ischemic stroke, iodinated contrast is frequently used for CTA and CTP imaging to provide information about the site of the vascular occlusion and the extent and severity of the ischemic

Received September 13, 2011; accepted after revision November 17.

From the Division of Neurology (M.D.I.V., L.K.C., F.L.S.), Department of Medicine, and Division of General Internal Medicine and Clinical Epidemiology and Women's Health Program (M.K.K.), University Health Network, University of Toronto, Toronto, Canada; UMC Utrecht Stroke Center (M.D.I.V.), Department of Neurology and Neurosurgery, Rudolf Magnus Institute of Neuroscience, UMC Utrecht, Utrecht, the Netherlands; Department of Health Policy, Management and Evaluation (M.K.K.), University of Toronto, Toronto, Ontario, Canada; Institute for Clinical Evaluative Sciences (J.F., M.S., A.R., M.K.K., F.L.S.), Toronto, Ontario, Canada; and the Canadian Stroke Network (M.K.K, F.L.S.).

This work was funded by an operating grant from the Canadian Stroke Network. The Registry of the Canadian Stroke Network is funded by an operating grant from the Ontario Ministry of Health and Long-Term Care. The Institute for Clinical Evaluative Sciences is supported by an operating grant from the Ontario Ministry of Health and Long-Term Care. The manuscript was reviewed and approved by the publications committee of the Registry of the Canadian Stroke Network. Dr Vergouwen was financially supported by an unrestricted grant from the Niels Stensen Foundation, the Netherlands. Dr Kapral is supported by a New Investigator Award from the Canadian Institutes for Health Research as well as career support from the Canadian Stroke Network and the University Health Network Women's Health Program. Dr Silver receives salary support from the Canadian Stroke Network.

The results and conclusions are those of the authors and should not be attributed to any of the sponsoring or funding agencies. The funding agencies had no role in the design or conduct of the study or the collection, management, analysis, or interpretation of the data.

Please address correspondence to Mervyn D.I. Vergouwen, MD, PhD, UMC Utrecht Stroke Center, Department of Neurology and Neurosurgery, Rudolf Magnus Institute of Neuroscience, UMC Utrecht, Heidelberglaan 100, 3584 CX Utrecht, the Netherlands; e-mail: m.d.i.vergouwen@umcutrecht.nl

Indicates open access to non-subscribers at www.ajnr.org

http://dx.doi.org/10.3174/ajnr.A3013 insult. This information is increasingly used as a tool in the decision to treat with either intravenous or intra-arterial thrombolytic therapy. ${ }^{1}$ In patients with intracerebral hemorrhage, CTA can be used to identify the presence of active contrast extravasation (the spot sign), which is a predictor of hematoma expansion. ${ }^{2}$ The spot sign might be used to select patients with intracerebral hemorrhage for early hemostatic therapy.

Because iodinated contrast can precipitate CIN and even acute renal failure in patients with unrecognized renal dysfunction, ${ }^{3}$ guidelines have been developed for the safe use of contrast agents. The Canadian Association of Radiologists recommends that risk assessment should be based on GFR rather than on the absolute level of serum creatinine. ${ }^{4}$ Patients with a GFR $>60 \mathrm{~mL} / \mathrm{min} / 1.73 \mathrm{~m}^{2}$ are at extremely low risk of developing CIN, those with a GFR of $30-60 \mathrm{~mL} / \mathrm{min} / 1.73 \mathrm{~m}^{2}$ have a low-to-moderate risk, and patients with severe renal dysfunction (defined as a GFR $<30 \mathrm{~mL} / \mathrm{min} / 1.73 \mathrm{~m}^{2}$ ) are at high risk. Nevertheless, in patients with acute stroke, contrast-enhanced studies are often performed without prior knowledge of kidney function, ${ }^{5,6}$ which places patients with pre-existent renal dysfunction at risk of further kidney damage. Our aim was to design a tool that helps clinicians identify patients with acute stroke with a high risk of CIN, in the absence of a recent GFR level, and to validate this prediction rule in another cohort of patients with stroke.

\section{Materials and Methods}

For this study, we used 2 cohorts of patients with stroke. The first cohort was the RCSN, Phase 3, which is a hospital-based registry of 33,119 consecutive patients presenting with a stroke or TIA within 14 
days after symptom onset to 11 regional stroke hospitals in Ontario, Canada, between July 1, 2003, and March 31, 2008. ${ }^{7,8}$ Approval for the use of the RCSN was obtained from the Research and Ethics Board at Sunnybrook Health Sciences Centre. Patients in this registry were identified prospectively, and data were abstracted systematically through chart review during the hospital stay and following hospital discharge by trained research nurses by using a standardized Case Report Form and custom electronic data-entry software that increases data quality. ${ }^{7}$ In addition, we used data from the 2002/2003 and 2004/2005 RCSN OSA, which is a random sample (containing 8574 patients) of all patients with stroke and TIA presenting to all 153 acute care hospitals in Ontario, Canada, evaluated biennially. ${ }^{9,10} \mathrm{Be}-$ cause the OSA cohort includes a random sample of patients in the RCSN regional stroke centers, patients who were in both cohorts were excluded from the OSA cohort. The prevalence of severe renal dysfunction was investigated in both cohorts. The RCSN Phase 3 cohort served as the derivation cohort for designing the prediction rule. For validation of this prediction rule, we used data from the OSA. For the current analysis, a research protocol with a statistical analysis plan was developed and submitted to the RCSN Publication Committee for approval.

\section{Data Collection and Variable Definitions}

From both cohorts, we identified all patients with an acute stroke or TIA who arrived within 5 hours of stroke onset, defined as a last seen normal time of $<5$ hours, because they might be eligible for an acute stroke intervention. Exclusion criteria were the following: 1) subarachnoid hemorrhage; 2) in-hospital stroke; 3) nonstroke; 4) unspecified stroke; 5) patients transferred from other emergency departments or acute hospitals; 6) known renal failure (on dialysis); 7) missing data on age, sex, or creatinine level; and 8) non-Ontario patient. Only first-stroke events in the data base were analyzed. We collected the following variables: age, sex, stroke type (ischemic stroke, TIA, or intracerebral hemorrhage), diabetes mellitus, hypertension, hyperlipidemia, smoking status, peripheral vascular disease, previous myocardial infarction, previous stroke/TIA, preadmission use of metformin or insulin, and creatinine level on admission. All variables were described in the Operational Manual of the RCSN using standard definitions. GFR was calculated by using the CKD-EPI equation (Table 1). ${ }^{11}$ Severe renal dysfunction was defined as chronic kidney disease stages 4 and 5 (GFR, 15-29 mL/min/1.73 $\mathrm{m}^{2}$ and $<15$ $\mathrm{mL} / \mathrm{min} / 1.73 \mathrm{~m}^{2}$, respectively). ${ }^{4,12}$

\section{Analyses}

Statistical analyses were performed by using a commercially available software package (SAS, Version 9.2 statistical software; SAS Institute, Cary, North Carolina). Baseline characteristics of both cohorts were summarized and compared by using descriptive statistics. In both cohorts, patients were divided into 2 groups: patients with severe renal dysfunction $\left(\mathrm{GFR}<30 \mathrm{~mL} / \mathrm{min} / 1.73 \mathrm{~m}^{2}\right.$ ) and patients with moderate-good renal function $\left(\mathrm{GFR} \geq 30 \mathrm{~mL} / \mathrm{min} / 1.73 \mathrm{~m}^{2}\right)$. Patients with severe renal dysfunction and moderate-good renal function were compared by using descriptive statistics. Categoric variables were analyzed by using the $\chi^{2}$ test. A $P$ value $<.05$ was considered statistically significant. Median values were presented with the IQR. In the RCSN cohort, a multivariable logistic regression analysis was performed to investigate which variables were independent predictors of severe renal dysfunction. Results of multivariable logistic regression analyses were presented as ORs with 95\% CIs. Variables that were found to be an independent predictor of severe renal dysfunction and that were

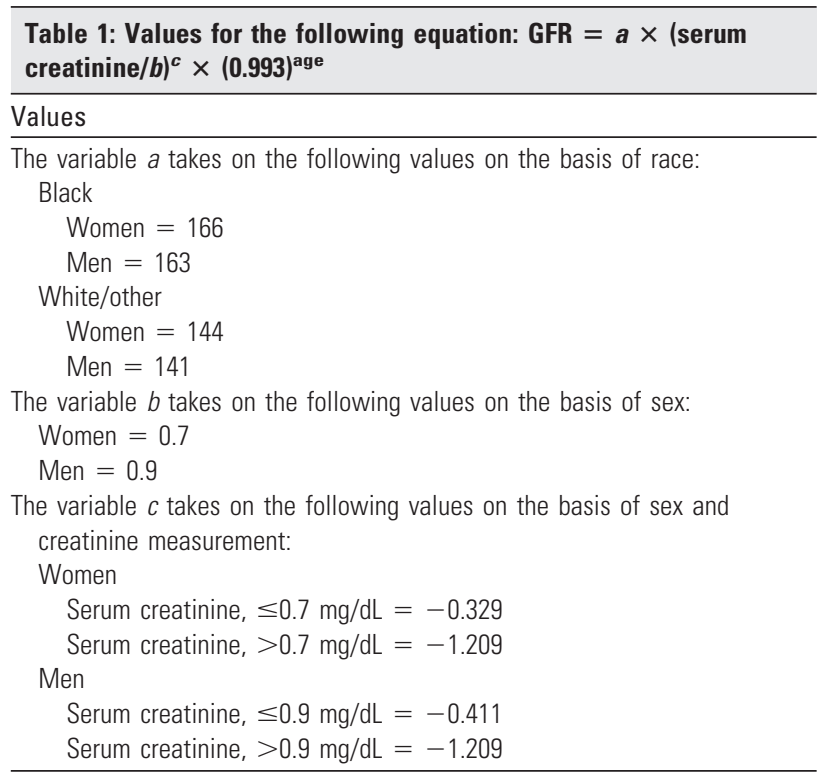

thought to be easy to evaluate in an emergency situation were used to develop a predictive tool to identify patients with severe renal dysfunction in the absence of the GFR.

From the logistic regression model, we used the regression coefficients for each risk factor that was determined to be predictive divided by the coefficient for age (as a reference), to provide a weighting factor for each variable. ${ }^{13,14}$ The presence of the predictive variables multiplied by the derived weighted factor allowed the calculation of a total number of points that would be used in a predictive tool for severe renal dysfunction, which was named the Renal Risk Score. The resulting numbers were rounded off to a number that can be divided by 5 , to improve the practicality of the score. Two models with different numbers of variables were created. We chose the model with superior c-stats for further analyses or, in case of similar c-statistics, the model with the lowest number of variables to improve the practicality of the score. The areas beneath the receiver operating characteristic curves for predicting severe renal dysfunction were compared in the derivation and validation cohorts. Then, we created the following Renal Risk Score categories arbitrarily: 10-60, 61-70, 71-80, 81-90, 91$100,101-110,111-120,121-130$. The prevalence of severe renal dysfunction was calculated for each category and compared in the derivation and validation cohorts with the use of $\chi^{2}$ statistics.

\section{Results}

\section{Comparison of the Cohorts}

Of the 33,119 patients in the RCSN Phase 3 cohort, 9872 met the study criteria (Fig 1). Of the 8574 patients in the OSA cohort, 2783 patients met the study criteria (Fig 1). Because 239 patients were included in both cohorts, we removed these patients from the OSA cohort, leaving 2544 patients. Baseline characteristics of both cohorts are described in Table 2. The 2 cohorts were comparable for sex, history of diabetes, myocardial infarction, and preadmission metformin and insulin use. In both cohorts, a majority of patients were older than 65 years of age and presented with either ischemic stroke or TIA.

\section{Incidence and Predictors of Severe Renal Dysfunction}

The overall prevalence of severe renal dysfunction was $4.9 \%$ (479 of 9872 patients) in the RCSN Phase 3 cohort and 5.2\% 


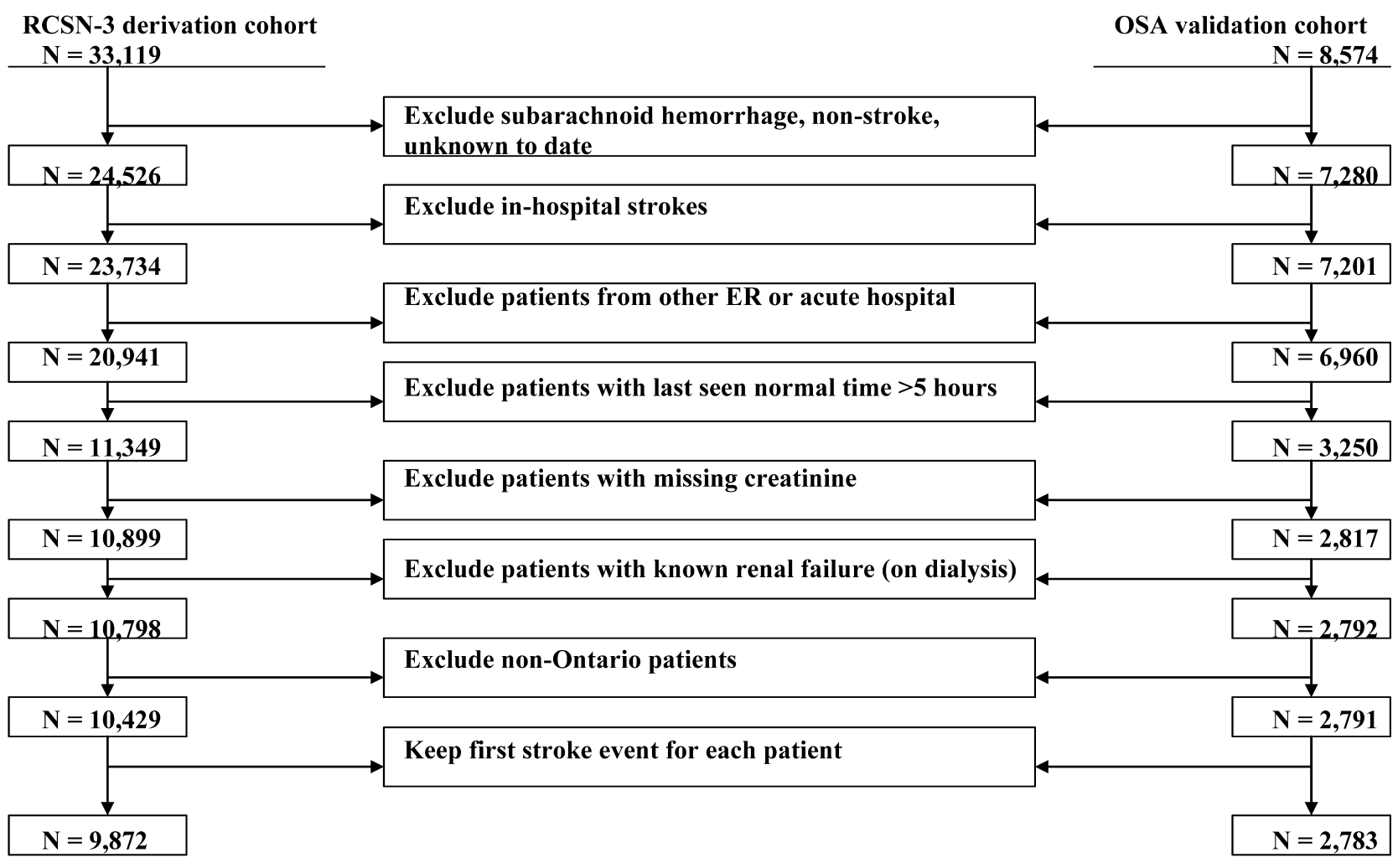

Fig 1. Cohort populations selection process.

\begin{tabular}{|c|c|c|c|c|c|c|c|c|c|}
\hline \multirow[b]{2}{*}{ Variable } & \multicolumn{4}{|c|}{ RCSN3 2003-2008 } & \multicolumn{4}{|c|}{ OSA $2002 / 03$ and $2004 / 05$} & \multirow{2}{*}{$\begin{array}{l}\text { Comparison of } \\
\text { all Patients from } \\
\text { RCSN versus } \\
\text { OSA ( } P \text { value) }\end{array}$} \\
\hline & $\begin{array}{c}\text { All } \\
(n=9872)\end{array}$ & $\begin{array}{l}\text { GFR } \geq 30 \\
(n=9393)\end{array}$ & $\begin{array}{l}\text { GFR }<30 \\
(n=479)\end{array}$ & $\begin{array}{c}P \\
\text { Value }\end{array}$ & $\begin{array}{c}\text { All } \\
(n=2544)\end{array}$ & $\begin{array}{l}\mathrm{GFR} \geq 30 \\
(n=2411)\end{array}$ & $\begin{array}{l}\text { GFR }<30 \\
(n=133)\end{array}$ & $\begin{array}{c}P \\
\text { Value }\end{array}$ & \\
\hline \multicolumn{10}{|l|}{ Age } \\
\hline Median (IOR) (yr) & 75 (65-83) & $74(64-82)$ & $82(77-88)$ & $<.0001$ & 77 (68-83) & 76 (67-83) & $82(77-87)$ & $<.0001$ & $<.0001$ \\
\hline$\geq 65$ yr (No.) (\%) & $7389(74.8)$ & $6936(73.8)$ & $453(94.6)$ & $<.0001$ & $2066(81.2)$ & $1936(80.3)$ & $130(97.7)$ & $<.0001$ & $<.0001$ \\
\hline No. of women (№.) (\%) & $4827(48.9)$ & $4827(48.9)$ & $294(61.4)$ & $<.0001$ & $1258(49.4)$ & $1177(48.8)$ & $81(60.9)$ & .007 & .62 \\
\hline Stroke type (No.) (\%) & & & & $<.0001$ & & & & .005 & $<.0001$ \\
\hline Ischemic stroke & $5496(55.7)$ & $5175(55.1)$ & $321(67.0)$ & & $1101(43.3)$ & $1026(42.6)$ & $75(56.4)$ & & \\
\hline TIA & 3307 (33.5) & 3176 (33.8) & $131(27.3)$ & & 1251 (49.2) & $1198(49.7)$ & $53(39.8)$ & & \\
\hline $\mathrm{ICH}$ & 1069 (10.8) & $1042(11.1)$ & $27(5.6)$ & & $192(7.5)$ & $187(7.8)$ & $\leq 5(\leq 3.8)$ & & \\
\hline Diabetes mellitus (o.) (\%) & $2178(22.1)$ & $2033(21.6)$ & $145(30.3)$ & $<.0001$ & $549(21.6)$ & $497(20.6)$ & $52(39.1)$ & $<.0001$ & .60 \\
\hline Hypertension (№.) (\%) & 6552 (66.4) & $6159(65.6)$ & $393(82.0)$ & $<.0001$ & $1512(59.4)$ & $1417(58.8)$ & $95(71.4)$ & .004 & $<.0001$ \\
\hline Hyperlipidemia (No.) (\%) & $3478(35.2)$ & $3298(35.1)$ & $180(37.6)$ & .27 & $718(28.2)$ & $684(28.4)$ & $34(25.6)$ & .48 & $<.0001$ \\
\hline $\begin{array}{l}\text { Peripheral vascular disease } \\
\text { (No.) (\%) }\end{array}$ & $529(5.4)$ & $483(5.1)$ & $46(9.6)$ & $<.0001$ & $105(4.1)$ & $92(3.8)$ & $13(9.8)$ & .0008 & .01 \\
\hline Current smoking (No.) (\%) & $1481(15.0)$ & $1450(15.4)$ & $31(6.5)$ & $<.0001$ & $313(12.3)$ & $304(12.6)$ & $9(6.8)$ & .046 & .0006 \\
\hline $\begin{array}{l}\text { Previous myocardial } \\
\text { infarction (No.) (\%) }\end{array}$ & $1492(15.1)$ & $1382(14.7)$ & $110(23.0)$ & $<.0001$ & $381(15.0)$ & $343(14.2)$ & $38(28.6)$ & $<.0001$ & .86 \\
\hline $\begin{array}{l}\text { Previous stroke or TIA } \\
\text { (No.) (\%) }\end{array}$ & $3357(34.0)$ & $3149(33.5)$ & $208(43.4)$ & $<.0001$ & $979(38.5)$ & $919(38.1)$ & $60(45.1)$ & 011 & $<.0001$ \\
\hline \multicolumn{10}{|l|}{$\begin{array}{l}\text { Preadmission medications } \\
\text { (No.) (\%): }\end{array}$} \\
\hline Metformin & $1038(10.5)$ & $1006(10.7)$ & $32(6.7)$ & .005 & $253(9.9)$ & $237(9.8)$ & $16(12.0)$ & .41 & .40 \\
\hline Insulin & $414(4.2)$ & $368(3.9)$ & $46(9.6)$ & $<.0001$ & $99(3.9)$ & 84 (3.5) & $15(11.3)$ & $<.0001$ & .49 \\
\hline
\end{tabular}

Note:- ICH indicates intracerebral hemorrhage.

(133 of 2544 patients) in the OSA cohort. Predictors of severe renal dysfunction were assessed in the RCSN Phase 3 cohort. Patients with severe renal dysfunction were older and more often women; more often presenting with ischemic stroke; more often had diabetes mellitus, hypertension, a previous myocardial infarction or stroke/TIA; more often were current smokers; and more often used metformin and insulin before admission. Results of the multivariable logistic regression analysis are shown in Table 3. In the RCSN derivation cohort, age, female sex, diabetes mellitus, hyperten- 


\begin{tabular}{lcccccc}
\hline \multicolumn{7}{l}{ Table 3: Results of multivariable logistic regression analyses using data from the RCSN (model 1) } \\
\hline Variable & Unit & OR $(95 \% \mathrm{Cl})$ & Estimate & $\beta$ Age & Estimate $\beta$ Age & Points Assigned \\
\hline Age (yr) & 1 & $1.07(1.06-1.08)$ & 0.0688 & 0.0688 & 1 & 1 (per yr) \\
Female sex & 1 & $1.27(1.04-1.54)$ & 0.2362 & 0.0688 & 3.44 & 5 \\
History of diabetes mellitus & 1 & $1.34(1.06-1.69)$ & 0.292 & 0.0688 & 0.25 & 13.46 \\
Preadmission insulin use & 1 & $2.52(1.74-3.67)$ & 0.9255 & 0.0688 & 9.05 & 15 \\
History of hypertension & 1 & $1.86(1.46-2.37)$ & 0.6219 & 0.0688 & & 10 \\
\hline
\end{tabular}

Table 4: Results of multivariable logistic regression analyses using data from the RCSN (model 2)

\begin{tabular}{|c|c|c|c|c|c|c|}
\hline Variable & Unit & OR $(95 \% \mathrm{CI})$ & Estimate & $\beta$ Age & Estimate $/ \beta$ Age & Points Assigned \\
\hline Age (yr) & 1 & $1.07(1.06-1.08)$ & 0.0685 & 0.0685 & 1 & 1 (per yr) \\
\hline Female sex & 1 & $1.32(1.09-1.61)$ & 0.2801 & 0.0685 & 4.09 & 5 \\
\hline History of diabetes mellitus & 1 & $1.31(1.03-1.66)$ & 0.2682 & 0.0685 & 3.92 & 5 \\
\hline Preadmission insulin use & 1 & $2.35(1.61-3.42)$ & 0.8545 & 0.0685 & 12.47 & 15 \\
\hline History of hypertension & 1 & $1.80(1.41-2.29)$ & 0.5866 & 0.0685 & 8.56 & 10 \\
\hline Previous myocardial infarction & 1 & $1.41(1.12-1.77)$ & 0.3425 & 0.0685 & 5.00 & 5 \\
\hline Peripheral vascular disease & 1 & $1.70(1.23-2.36)$ & 0.5313 & 0.0685 & 7.75 & 10 \\
\hline
\end{tabular}

\begin{tabular}{|c|c|c|c|}
\hline $\begin{array}{l}\text { Table 5: Pre } \\
\text { Score categ }\end{array}$ & $\begin{array}{l}\text { nce of renal insuffi } \\
s\end{array}$ & cy according to & al Risk \\
\hline Renal Risk & Severe Ren & ysfunction (GFR & \\
\hline Score Cutoff & RCSN3 $(n / M)(\%)$ & OSA $(n / M)(\%)$ & $P$ Value \\
\hline $10-60$ & $\leq 5 / 981(\leq 0.005)$ & $\leq 5 / 196(\leq 0.03)$ & .84 \\
\hline $61-70$ & $7 / 1011(0.7)$ & $\leq 5 / 210(\leq 0.02)$ & .72 \\
\hline $71-80$ & $34 / 1622(2.1)$ & $10 / 457(2.2)$ & .90 \\
\hline 81-90 & $91 / 2488(3.7)$ & $33 / 731$ (4.5) & .29 \\
\hline 91-100 & $180 / 2519(7.1)$ & $44 / 665(6.6)$ & .64 \\
\hline $101-110$ & $132 / 1055(12.5)$ & $33 / 249$ (13.3) & .75 \\
\hline $111-120$ & 29/182 (15.9) & 9/32 (28.1) & .10 \\
\hline $121-130$ & - & - & .13 \\
\hline
\end{tabular}

Note:- - indicates small cell number $(\leq 5)$.

sion, insulin use, previous myocardial infarction, and peripheral vascular disease were independent predictors of severe renal dysfunction.

\section{Renal Risk Score}

On the basis of the results of the multivariable logistic regression analysis in the RCSN derivation cohort, 2 models were created. The first model included the following variables: age, sex, hypertension, diabetes mellitus, and insulin use (Table 3 ). In the second model, we also included a history of myocardial infarction and peripheral vascular disease (Table 4$)$. The areas under the curve were similar in both models $(0.73$ and 0.74 , respectively). The first model with 5 items was selected for further analyses.

The Renal Risk Score was designed as follows: (age in years) $+(5$ points for women $)+(5$ points for history of diabetes mellitus $)+(15$ points for preadmission insulin use $)+(10$ points for history of hypertension). In all Renal Risk Score categories, no significant differences were observed for the prevalence of severe renal dysfunction in the OSA cohort compared with the RCSN cohort (Table 5 and Fig 2). There was no significant difference $(P=.66)$ in the area under the receiver operating characteristic curves between the RCSN Phase 3 derivation cohort (OR, 1.07 [95\% CI, 1.06-1.08]) (Fig 3A) and the OSA validation cohort (OR, 1.07 [95\% CI, 1.05-1.09]) (Fig 3B). The prevalence of severe renal dysfunction is negligible in patients with a Renal Risk Score of $\leq 70$ but increases in patients with higher Renal Risk Scores (Table 5 and Fig 2).
For example, in patients with a Renal Risk Score of 71-80, the prevalence in the RCSN Phase 3 and OSA cohort was $2.1 \%$ and $2.2 \%$, respectively; in patients with scores $91-100$, it was $7.1 \%$ and $6.6 \%$; and in those with scores $111-120$, it was $15.9 \%$ and $28.1 \%$. No data are presented for patients with a Renal Risk Score of 121-130 because of small cell numbers.

\section{Discussion}

The results of the present study show that the prevalence of severe renal dysfunction in patients with acute stroke is approximately $5 \%$, and that the Renal Risk Score is an easy tool to identify patients with acute stroke at high risk of CIN. The prevalence of severe renal dysfunction is negligible if the Renal Risk Score is $<70$ but increases with higher Renal Risk Scores.

Several studies investigated CIN in patients with stroke who received contrast-enhanced CT. The incidence of acute nephropathy was found to be $2.9 \%-6 \%$, and of renal replacement therapy related to contrast-enhanced imaging, $<1 \%{ }^{3,5,15,16}$ However, all these studies were retrospective ones in which renal function was often known at the moment of scanning. As a result, it may be that clinical judgment was used to withhold contrast-enhanced imaging from those at highest risk of CIN. Thus, the risk of CIN might be much higher if GFR results were not known before contrast-enhanced imaging was performed.

The Renal Risk Score can be calculated with parameters that can be easily determined after a brief initial clinical assessment (eg, medications, presence of hypertension, diabetes). Patients on chronic renal dialysis were excluded from the cohorts because it is easy to determine that these patients have renal failure. In patients not on dialysis, the Renal Risk Score is an easy tool to assess the risk of severe renal dysfunction at baseline. In patients with a Renal Risk Score $<70$, the risk of severe renal dysfunction at baseline is negligible. Thus, in those patients, the GFR determination does not have to be available before the use of contrast. Higher Renal Risk Scores should be used as a cautionary guide, not an absolute contraindication to giving contrast. The final decision of whether to give contrast or to wait for a serum creatinine level should be based on the perceived clinical need for angiographic and cerebral perfusion studies versus the risk of developing CIN. If the decision is to give contrast before the GFR result is avail- 


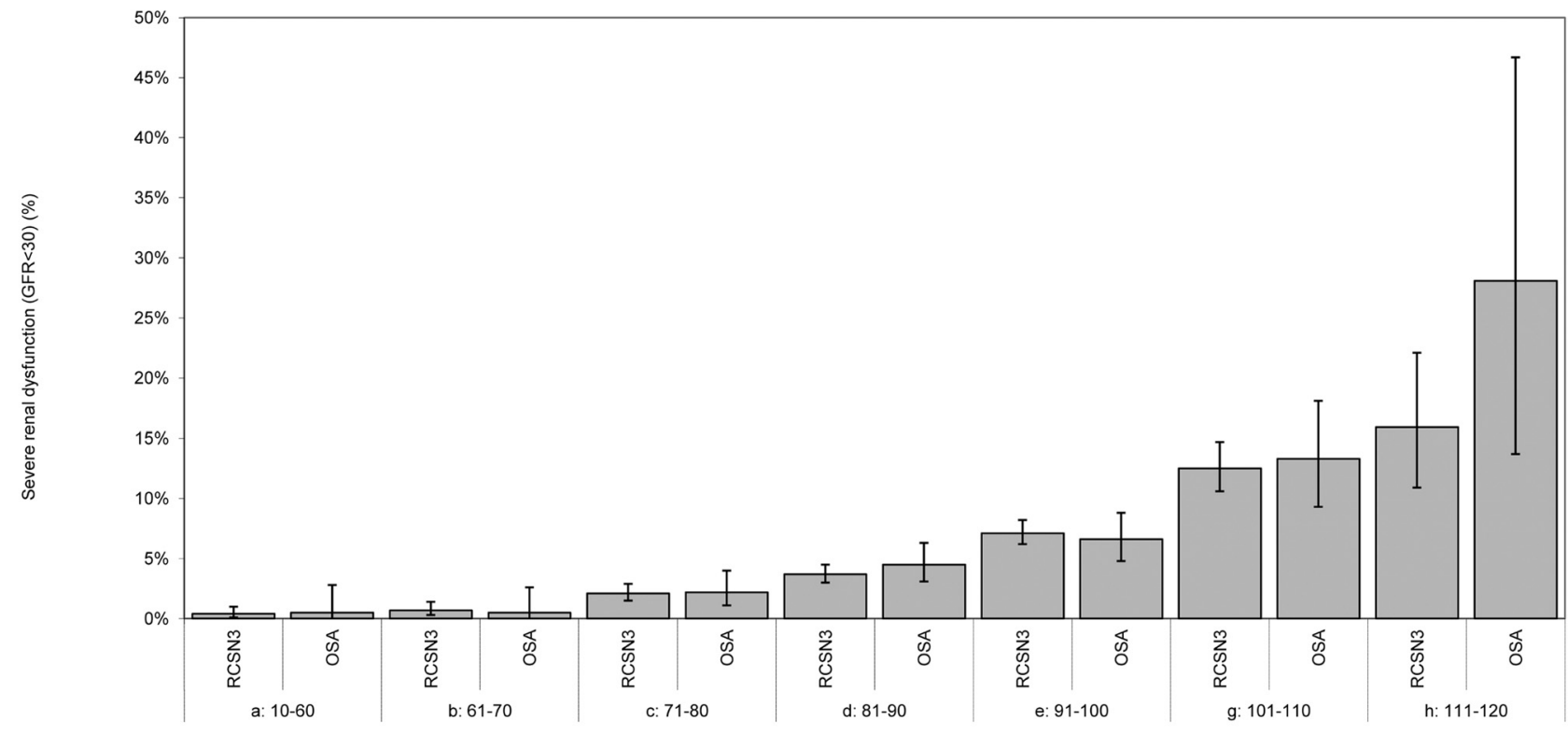

Risk score groups

Fig 2. Comparison of severe renal dysfunction in the derivation and validation cohorts according to Renal Risk Score categories.

RCSN-3: AUC $=0.73$

Hosmer-Lemeshow test: $p=0.60$

A

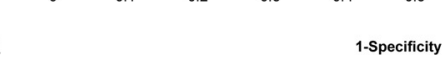

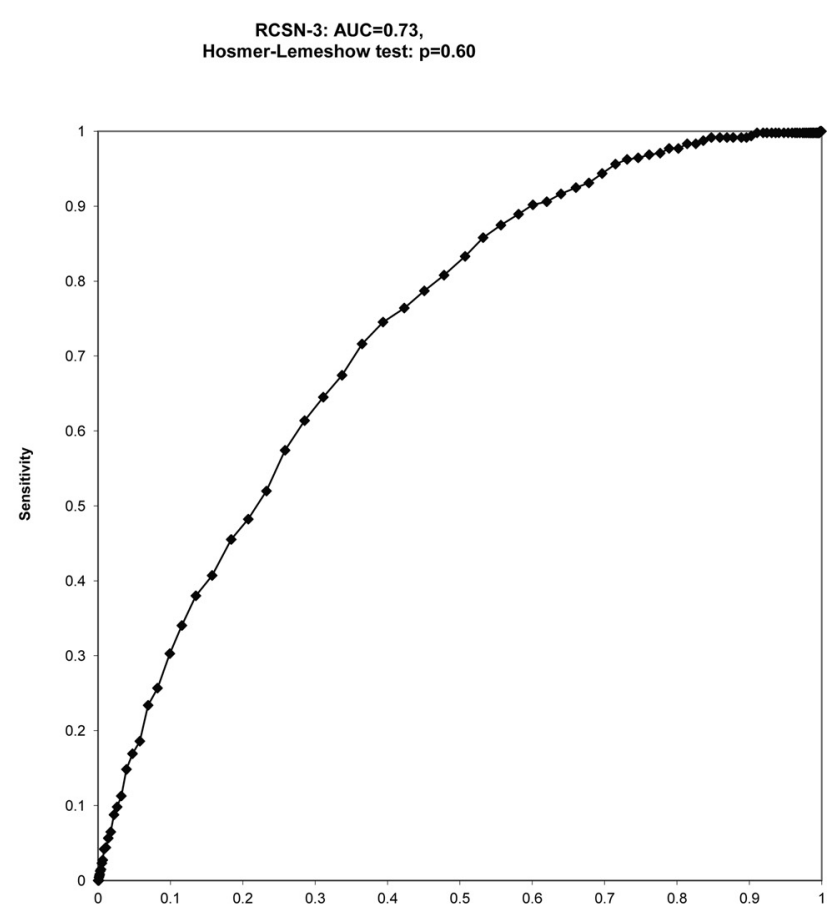

Fig 3. A, Receiver operating characteristic curve for predicting severe renal dysfunction in the RCSN cohort (model 1). B, Receiver operating characteristic curve for predicting severe renal dysfunction in the OSA cohort.

able, we still recommend that the preimaging GFR should be reviewed as soon as possible after a contrast-enhanced CT scan is performed. Even patients with low Renal Risk Scores have a small chance of having unrecognized severe renal dysfunction. In addition to using the Renal Risk Score to better select patients for contrast-enhanced imagining, other measures should be implemented to prevent CIN, including administer- ing the lowest possible volume of a low or iso-osmolar contrast agent and keeping the patient well hydrated.

The Renal Risk Score predicts the presence of severe renal dysfunction and not necessarily the development of CIN. To design a tool that predicts the development of CIN, one would have to administer iodinated contrast to a large number of consecutive patients with acute stroke, regardless of their GFR 
level or presumed risk of renal disease. Such a study would be unethical to perform because a considerable number of patients might develop renal failure. Therefore, we decided to design a rule that predicts the presence of severe renal dysfunction, because this group has the highest risk of developing CIN. ${ }^{4}$ Most of our patients had noncontrast brain imaging acutely, and contrast-enhanced imaging was considered only after checking the GFR. For derivation of the Renal Risk Score, all patients presenting within $<5$ hours from stroke onset were included in the analysis regardless of whether they had a CT with contrast or not, so the score should have broad applicability.

In clinical practice, a GFR $<30 \mathrm{~mL} / \mathrm{min} / 1.73 \mathrm{~m}^{2}$ is considered an absolute contraindication for administration of iodinated contrast. Therefore, we designed a score that calculates the risk of having a GFR $<30$. We considered designing a score that calculates the risk of having a GFR $<60$; however, patients with a GFR of 30-60 have a much lower risk of CIN compared with patients with a GFR $<30$. Furthermore, prehydration prophylaxis has been shown to be effective in preventing CIN in patients with a GFR of 30-60 but not in patients with a GFR $<30 .{ }^{17}$ Finally, we only did a "narrow" validation of the Renal Risk Score in a population that is similar to the derivation cohort. Future studies should be done to validate this prediction rule in other cohorts of patients with stroke.

\section{Conclusions}

Severe renal dysfunction is not uncommon in patients with acute stroke. The Renal Risk Score provides a simple tool that uses 5 simple clinical items to help clinicians identify patients who are likely to have severe renal dysfunction and are at high risk of developing CIN. Given the importance of initiating stroke interventions as soon as possible after stroke onset, the hope is that using this prediction tool will avoid unnecessary delays related to waiting for laboratory results in patients needing brain and vascular imaging studies that require iodinated contrast.

Disclosures: Leanne K. Casaubon—UNRELATED: Payment for Lectures (including service on Speakers Bureaus): Sanofi-Aventis. Frank Silver-UNRELATED: Board Membership: Boehringer Ingelheim Canada, Bayer Inc. Canada, Comments: stroke advisory boards, Payment for Lectures (including service on Speakers Bureaus). Boehringer Ingelheim Canada, Servier Canada, Comments: Speaker.

\section{References}

1. Amenta PS, Ali MS, Dumont AS, et al. Computed tomography perfusionbased selection of patients for endovascular recanalization. Neurosurg Focus 2011;30:E6

2. Wada R, Aviv RI, Fox AJ, et al. CT angiography “spot sign” predicts hematoma expansion in acute intracerebral hemorrhage. Stroke 2007;38:1257-62

3. Josephson SA, Dillon WP, Smith WS. Incidence of contrast nephropathy from cerebral CT angiography and CT perfusion imaging. Neurology 2005;64:1805-06

4. Benko A, Fraser-Hill M, Magner P, et al, for the Canadian Association of Radiologists. Canadian Association of Radiologists: consensus guidelines for the prevention of contrast-induced nephropathy. Can Assoc Radiol J 2007;58:79-87

5. Krol AL, Dzialowski I, Roy J, et al. Incidence of radiocontrast nephropathy in patients undergoing acute stroke computed tomography angiography. Stroke 2007;38:2364-66

6. Smith WS, Roberts HC, Chuang NA, et al. Safety and feasibility of a CT protocol for acute stroke: combined CT, CT angiography, and CT perfusion imaging in $\mathbf{5 3}$ consecutive patients. AJNR Am J Neuroradiol 2003;24:688-90

7. Silver FL, Kapral MK, Lindsay MP, et al. International experience in stroke registries: lessons learned in establishing the Registry of the Canadian Stroke Network. Am J Prev Med 2006;31:S235-37

8. Kapral MK, Laupacis A, Phillips SJ, et al, for the Investigators of the Registry of the Canadian Stroke Network. Stroke care delivery in institutions participating in the Registry of the Canadian Stroke Network. Stroke 2004;35:1756-62. Epub 2004 May 13

9. Kapral MK, Lindsay MP, Silver FL, et al. Registry of the Canadian Stroke Network: Report on the 2002/03 Ontario Stroke Audit. Toronto, Ontario, Canada: Institute for Clinical Evaluative Sciences; 2006

10. Kapral MK, Hall RE, Silver FL, et al. Registry of the Canadian Stroke Network: Report on the 2004/05 Ontario Stroke Audit. Toronto, Ontario, Canada: Institute for Clinical Evaluative Sciences; 2009

11. Levey AS, Stevens LA, Schmid CH, et al. CKD-EPI (Chronic Kidney Disease Epidemiology Collaboration): a new equation to estimate glomerular filtration rate. Ann Intern Med 2009;150:604-12

12. National Kidney Foundation. K/DOQI clinical practice guidelines for chronic kidney disease: evaluation, classification, and stratification. Am J Kidney Dis 2002;39:S1-266

13. Fine MJ, Auble TE, Yealy DM, et al. A prediction rule to identify low-risk patients with community-acquired pneumonia. $N$ Engl $J$ Med 1997;336:243-50

14. Lee DS, Austin PC, Rouleau JL, et al. Predicting mortality among patients hospitalized for heart failure: derivation and validation of a clinical model. JAMA 2003;290:2581-87

15. Hopyan JJ, Gladstone DJ, Mallia G, et al. Renal safety of CT angiography and perfusion imaging in the emergency evaluation of acute stroke. AJNR Am J Neuroradiol 2008;29:1826-30

16. Oleinik A, Romero JM, Schwab K, et al. CT angiography for intracerebral hemorrhage does not increase risk of acute nephropathy. Stroke 2009;40:2393-97

17. Chong E, Poh KK, Liang S, et al. Comparison of risks and clinical predictors of contrast-induced nephropathy in patients undergoing emergency versus nonemergency percutaneous coronary interventions. J Interv Cardiol 2010;23: 451-59 